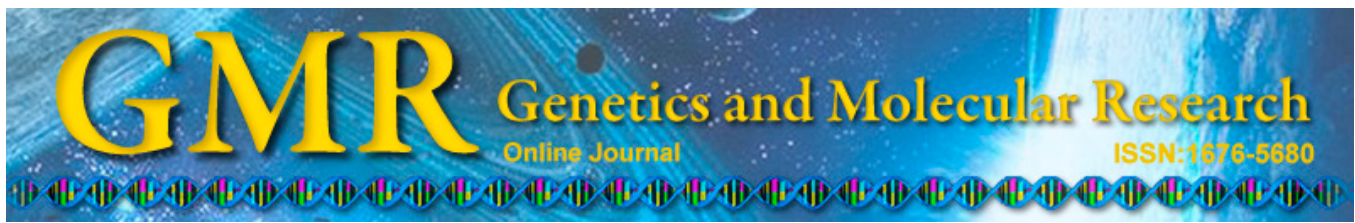

\title{
Association between primary open angle glaucoma and genetic polymorphisms GSTM1/GSTT1 in patients from Goiânia Central-West Region of Brazil
}

\author{
C.T.X.Silva ${ }^{1,2}$, N.B. Costa ${ }^{1,2}$, K.S.F. Silva ${ }^{1,2}$, R.E. Silva ${ }^{1,3}$ and K.K.V.O. Moura ${ }^{1,2}$ \\ ${ }^{1}$ Programa de Pós-Graduação em Genética, \\ Pontifícia Universidade Católica de Goiás, Goiânia, GO, Brasil \\ ${ }^{2}$ Núcleo de Pesquisas Replicon, Departamento de Biologia, \\ Pontifícia Universidade Católica de Goiás, Goiânia, GO, Brasil \\ ${ }^{3}$ Pronto Clínica de Olhos, Goiânia, GO, Brasil \\ Corresponding author: K.K.V.O. Moura \\ E-mail: kkverolli@pucgoias.edu.br
}

Genet. Mol. Res. 13 (4): 8870-8875 (2014)

Received September 30, 2013

Accepted June 17, 2014

Published October 31, 2014

DOI http://dx.doi.org/10.4238/2014.October.31.2

ABSTRACT. In this study, we evaluated the genotype profile of
GSTM1 and GSTT1 polymorphisms in patient carriers of primary
open-angle glaucoma in the population of Goiânia, GO, Brazil. This
case-control study included 100 Brazilian patients with glaucoma and
53 patients without glaucoma. Blood samples were genotyped for
polymorphisms in GST genes using polymerase chain reaction-based
methods. Polymorphism frequencies were compared using the $\chi^{2}$ test
and odds ratio ( $\alpha=0.05$ ). The GSTM1-present genotype was $40 \%$ in
the glaucoma group and $71.6 \%$ in the control group, while the GSTM1
null genotype was 60 and $28.3 \%$ in the same groups, respectively.
The GSTT1-present genotype was $52 \%$ in the primary open-angle
glaucoma group and $66 \%$ in the control group; the null genotype was
$48 \%$ in the case group and $34 \%$ in the control group. The GSTM1 null 
genotype was more frequent in the glaucoma group than in the control group $(\mathrm{P}=0.0004$; odds ratio $=6.7 ; 95 \%$ confidence interval $=2.7$ 20.3). The combined GSTM1 null and GSTT1-present genotypes were more frequent in the primary open-angle glaucoma group compared to the control group $(\mathrm{P}=0.02$; odds ratio $=3.1 ; 95 \%$ confidence interval $=1.2-7.9$ ).

Key words: Glaucoma; Glutathione S-transferase; GSTM1; GSTT1

\section{INTRODUCTION}

Primary open-angle glaucoma (POAG) is a progressive optic, chronic, and multifactorial neuropathy characterized by the loss of optic nerve fibers. The disease develops through openings in the anterior chamber of the eye, leading to abnormalities in the visual field (Saccà et al., 2005; Abu-Amero et al., 2008).

POAG is a concern for physicians, patients, and authorities who seek methods for preventing blindness from this disease. It is the second leading cause of blindness worldwide with a high incidence according to the Brazilian Consensus of Primary Open-Angle Glaucoma (Paranhos-Júnior et al., 2009). However, the early development of POAG is not fully understood. The disease is asymptomatic and is rarely a target of prevention campaigns. POAG is frequently diagnosed incidentally during ophthalmic examinations for other ocular dysfunctions (Mello and Mandía-Junior, 2005).

Several studies identified an association between POAG and glutathione S-transferase (GST) polymorphisms (Juronen et al., 2000; Yildirim et al., 2005; Ünal et al., 2007; Rocha et al., 2011). GSTs are found in a variety of ocular structures including the aqueous humor, ciliary body, and crystalline lens. The production and release of toxic metabolites may induce changes in the structures of proteins present in the aqueous humor and trabecular meshwork, leading to reduced flow and increased intraocular pressure (Izzotti et al., 2006).

Polymorphisms identified in GSTM1 include GSTM1*0, GSTM1*A, and GSTM1*B. GSTM1* 0 is deleted, and homozygotes (GSTM1 null genotype) express no protein. GSTM1*A and $G S T M 1 * B$ differ by a single base, and the catalytic effectiveness of the enzymes encoded by these alleles is similar (Sprenger et al., 2000). There are 2 theta-class genes, GSTT1 and GSTT2, located on chromosome 22.6. GSTT1 is represented by 2 alleles: a functional or wildtype allele $\left(G S T T 1^{*} 1\right)$ and a nonfunctional or null allele $(G S T T 1 * 0)$. Studies showed that the GSTT1*0 allele corresponds to total or partial deletion of the gene, causing a deficiency in enzymatic activity (Ünal et al., 2007).

Oxidative stress and antioxidant systems are very important in ocular tissue, which regenerates slowly, increasing the risk of toxin accumulation and resulting in tissue damage. Additionally, molecular changes in these systems contribute to the development of glaucoma, cataract, and other age-related diseases (Yildirim et al., 2005). The ocular epithelia express genes that encode for GSTs. Among the cytosolic proteins, GSTM1 and GSTT1 show the highest correlation with glaucoma (Ünal et al., 2007).

In this study, we evaluated the genotypic profile of GSTM1 and GSTT1 polymorphisms in patients who were carriers of POAG in the city of Goiânia. 


\section{MATERIAL AND METHODS}

We conducted a case-control study of 100 glaucoma patients. The control group contained 53 patients with normal eye exams that were within reference standards. The patients were treated at the Pronto Eye Clinic in Goiânia. All patients underwent ophthalmologic examination, including measurement of intraocular pressure, visual acuity test, automated perimeter test, gonioscopy, examination of the optic disc, and retinal exam. The project was approved by the National Ethics Commission in Research/National System of Information about Ethics in Research involving Human Subjects CEP/PUC-GOIÁS (FR160294), and informed consent was obtained from all patients.

Genomic DNA was extracted from peripheral blood according to the instructions in the commercial kit Illustra Blood Genomic Prep Mini Spin ${ }^{\circledR}$ (GE Healthcare, Little Chalfont, UK). Allele-specific polymerase chain reaction (PCR) of the DNA was performed in triplicate. Primer sequences for the GSTM1 and GSTT1 genes have been reported previously by Abdel-Rahman et al. (1996). PCR was carried out according to the protocol proposed by Frare (2011). As an internal control for the presence of human DNA and to avoid false-negatives, we used the zinc-finger gene $Z F X / Z F Y$ gene, which amplifies specific sequences on sex chromosomes (Arruda et al., 2008). One or 2 copies of this gene were classified as genotype-present, while those with homozygous deletions were classified as genotype-null.

We used the statistical analysis BioEstat ${ }^{\mathbb{}}$ software (version 5.0; Ayres et al., 2007) to analyze the results; frequencies were determined using the chi-square test and odds ratio (OR).

\section{RESULTS}

The POAG group included 46 women and 54 men (average age of $60.5 \pm 18.8$ years old), while 36 women and 22 men $(44.7 \pm 16.4$ years $)$ were included in the control group $(\mathrm{P}<0.0001)$.

Table 1 shows the GSTM1 and GSTT1 genotype frequencies for the experimental and control groups. In the glaucoma group, the GSTM1 gene was present in $40 \%$ of patients. However, the GSTM1 gene was detected in $72 \%$ of patients in the control group. The frequency of the GSTM1 null gene in the glaucoma group was 2.12-fold higher than in the control group. The GSTT1 gene was present in 52\% of patients in the experimental group. In the control group, $66 \%$ of patients possessed the GSTT1 gene, while $34 \%$ of patients were GSTT1 null. The frequency of GSTT1 null in the glaucoma group was 1.4-fold higher compared to the control group.

Table 1. GSTM1 and GSTT1 genotypic frequency in primary open-angle glaucoma (POAG) and control group.

\begin{tabular}{|c|c|c|c|c|c|c|}
\hline \multirow[t]{2}{*}{ Genotype } & \multicolumn{2}{|c|}{ POAG } & \multicolumn{2}{|c|}{ Control } & \multirow[t]{2}{*}{ OR $(95 \% \mathrm{CI})$} & \multirow[t]{2}{*}{$\mathrm{P}$} \\
\hline & $\%$ & $\mathrm{~N}$ & $\%$ & $\mathrm{~N}$ & & \\
\hline \multicolumn{7}{|l|}{ GSTM1 } \\
\hline Present & 40 & 40 & 72 & 38 & $0.3(0.1-0.5)$ & $0.0004^{*}$ \\
\hline Null & 60 & 60 & 28 & 15 & & \\
\hline Total & 100 & 100 & 100 & 53 & & \\
\hline \multicolumn{7}{|l|}{ GSTT1 } \\
\hline Present & 52 & 52 & 66 & 35 & $0.6(0.3-1.1)$ & 0.13 \\
\hline Null & 48 & 48 & 34 & 18 & & \\
\hline Total & 100 & 100 & 100 & 53 & & \\
\hline
\end{tabular}

$\mathrm{POAG}=$ primary open-angle glaucoma. $*$ Statistically significant value. 


\begin{tabular}{|c|c|c|c|c|c|c|c|}
\hline \multirow[t]{2}{*}{ GSTMI } & \multirow[t]{2}{*}{ GSTT1 } & \multicolumn{2}{|c|}{ Experimental } & \multicolumn{2}{|c|}{ Control } & \multirow[t]{2}{*}{ OR $(95 \% \mathrm{CI})$} & \multirow[t]{2}{*}{$\mathrm{P}$} \\
\hline & & $\%$ & $\mathrm{~N}$ & $\%$ & $\mathrm{~N}$ & & \\
\hline Present & Present & 23 & 23 & 47 & 25 & $1.0^{1}$ & (Reference) \\
\hline Present & Null & 17 & 17 & 24 & 13 & $1.4(0.5-3.5)$ & 0.60 \\
\hline Null & Present & 29 & 29 & 19 & 10 & $3.1(1.2-7.9)$ & $0.02 *$ \\
\hline Null & Null & 31 & 31 & 10 & 5 & $6.7(2.7-20.3)$ & $0.0007^{*}$ \\
\hline Total & & 100 & 100 & 100 & 53 & & \\
\hline
\end{tabular}

*Statistically significant. ${ }^{1}$ Reference group, individuals with genotype at low risk (GSTM1 and GSTT1 presence).

\section{DISCUSSION}

GSTs are found in many different ocular structures, including the aqueous humor, ciliary body, and crystalline lens (Juronen et al., 2000; Yildirim et al., 2005; Ünal et al., 2007; Rocha et al., 2011). Toxic metabolites may induce changes in the protein structures present in the aqueous humor and trabecular meshwork, leading to reduced flow and increased intraocular pressure (Izzotti et al., 2006). The eye requires an efficient, enzymatically driven detoxification system. Epidemiological studies suggest that the susceptibility to ocular pathologies are correlated with the GST system (Juronen et al., 2000; Yildirim et al., 2005).

The association GSTM1-null and GSTT1-present genotypes increase the risk of developing glaucoma by 3.1 -fold. In combination, the null genotypes GSTM1/GSTT1 increase the risk of developing glaucoma by 6.7-fold. Izzotti et al. (2003) analyzed an Italian population and found a higher frequency of GSTM1-null in glaucoma patients compared to healthy patients; this was the first association identified between GSTM1 polymorphisms and POAG. However, the GSTT1 genotype was not statistically significant compared to the other groups, which agrees with our results.

Studies performed in the Turkish population by Yildirim et al. (2005) also agree with our results. A higher frequency of GSTM1-null genotype was found among glaucoma patients compared to controls $(54.9 \mathrm{vs} 40.9 \%$; OR $=1.64 ; 95 \% \mathrm{CI}=1.10-2.59)$, and the frequency of GSTT1 in both experimental and control groups was not statistically significant.

Abu-Amero et al. (2008) studied a population in Saudi Arabia and reported an association between the GSTM1/GSTT1 null genotypes and POAG. Their results agree with ours and indicate that decreased GST function interferes with oxidative metabolism, leading to the optic nerve damage caused by oxidative stress; the GST polymorphism may be a risk factor for the development of POAG.

Other studies in the Brazilian population from Bahia, performed by Rocha et al. (2011), confirm our findings in the Goiás population. They reported that the GSTM1-null polymorphism was significantly more frequent in the POAG group than in the control group.

However, Juronen et al. (2000) analyzed the Estonia population and Ünal et al. (2007) analyzed the Turkish population and reported that the GSTM1-present genotype was associated with an increased risk of POAG development compared with controls. These findings differ from those observed in our study.

Studies examining autoimmunity revealed a relationship between the GSTM1-present genotype and glaucoma. Yang et al. (2001) showed that a GST antigen was present in 52\% of patients with glaucoma and in $20 \%$ of patients in the control group $(\mathrm{P}=0.05)$. Patients showed higher levels of anti-GST compared with control groups. Their results suggest that individu- 
als expressing GSTM1 are at an increased risk of developing auto-antibodies against GST and have an increased risk of glaucoma.

In contrast, Jansson et al. (2003) studied a Swedish population and detected no significant association between POAG and GST polymorphisms.

We previously studied the current group of patients and controls and found no association between the polymorphism p53 codon 72 (ARG/PRO) and POAG (Silva et al., 2009). Costa (2012) observed a statistically significant association $(P<0.0001)$ between the CYP1A1m1 polymorphism and and POAG.

Our study is one of the firsts to describe the GST polymorphism in the Central-West region of Brazil. We identified a statistically significant association between the GSTM1-null genotype and POAG.

Genetic studies are important for determining the mechanisms leading to the development of glaucoma (Hogewind et al., 2007). New diagnostic methods and treatments are also important. The correlation between genotype and phenotype allows the observation of different clinical manifestations as well as therapeutic responses to glaucoma. Understanding the genetic factors involved in glaucoma will contribute to earlier diagnosis and the prevention of glaucomatous damage using both conventional treatment and even gene therapy (LopezMartinez et al., 2007; Kwon et al., 2009; Rasool et al., 2010). In the future, it may be possible to prevent glaucoma through genetic counseling. Understanding the genetics of glaucoma is essential for decreasing disease risk. Our results may contribute to individual medical monitoring for patients with glaucoma and their families.

\section{REFERENCES}

Abdel-Rahman SZ, el-Zein RA, Anwar WA and Au WW (1996). A multiplex PCR procedure for polymorphic analysis of GSTM1 and GSTT1 genes in population studies. Cancer Lett. 107: 229-233.

Abu-Amero KK, Morales J, Mohamed GH, Osman MN, et al. (2008). Glutathione S-transferase M1 and T1 polymorphisms in Arab glaucoma patients. Mol. Vis. 14: 425-430.

Arruda JT, Silva DM, Silva CC, Moura KK, et al. (2008). Homologous recombination between HERVs causes duplications in the AZFa region of men accidentally exposed to cesium-137 in Goiania. Genet. Mol. Res. 7: 1063-1069.

Ayres M, Ayres-Júnior M, Ayres DL and Santos AAS (2007). BioEstat-Aplicações Estatísticas nas Áreas das Ciências Bio-Médicas. 5th edn., Belém.

Costa NB (2012). Análise do Polimorfismo CYP1A1M1 em Pacientes com Glaucoma Primário de Ângulo Aberto (Master's thesis). Pontifícia Universidade Católica de Goiás, Goiânia.

Frare AB (2011). Investigação dos Polimorfismos GSTM1 e GSTT1 em Mulheres com Endometriose (Master's thesis). Pontifícia Universidade Católica de Goiás, Goiânia.

Hogewind BF, Gaplovska-Kysela K, Theelen T, Cremers FP, et al. (2007). Identification and functional characterization of a novel MYOC mutation in two primary open angle glaucoma families from The Netherlands. Mol. Vis. 13: 1793-1801.

Izzotti A, Sacca SC, Cartiglia C and De Flora S (2003). Oxidative deoxyribonucleic acid damage in the eyes of glaucoma patients. Am. J. Med. 114: 638-646.

Izzotti A, Bagnis A and Sacca SC (2006). The role of oxidative stress in glaucoma. Mutat. Res. 612: 105-114.

Jansson M, Rada A, Tomic L, Larsson LI, et al. (2003). Analysis of the Glutathione S-transferase M1 gene using pyrosequencing and multiplex PCR - no evidence of association to glaucoma. Exp. Eye Res. 77: 239-243.

Juronen E, Tasa G, Veromann S, Parts L, et al. (2000). Polymorphic glutathione S-transferase M1 is a risk factor of primary open-angle glaucoma among Estonians. Exp. Eye Res. 71: 447-452.

Kwon YH, Fingert JH, Kuehn MH and Alward WL (2009). Primary open-angle glaucoma. N. Engl. J. Med. 360: 1113-1124.

Lopez-Martinez F, Lopez-Garrido MP, Sanchez-Sanchez F, Campos-Mollo E, et al. (2007). Role of MYOC and OPTN sequence variations in Spanish patients with primary open-angle glaucoma. Mol. Vis. 13: 862-872.

Mello PAA and Mandía-Junior C (2005). II Consenso Brasileiro de Glaucoma Primário de Ângulo Aberto. Sociedade Brasileira de Glaucoma, São Paulo. 
Paranhos-Júnior A, Omi CA and Prata-Júnior JÁ (2009). III Consenso Brasileiro de Glaucoma Primário de Ângulo Aberto. Sociedade Brasileira de Glaucoma, São Paulo.

Rasool HAA, Nowier SR, Gheith M, Saif ATS, et al. (2010). The risk of primary open angle glaucoma and glutathione S transferase M1 and T1 polymorphism among Egyptians. J. Am. Sci. 6: 375-381.

Rocha AV, Talbot T, Magalhães da Silva T, Almeida MC, et al. (2011). Is the GSTM1 null polymorphism a risk factor in primary open angle glaucoma? Mol. Vis. 17: 1679-1686.

Saccà SC, Pascotto A, Camicione $\mathrm{P}$, Capris $\mathrm{P}$, et al. (2005). Oxidative DNA damage in the human trabecular meshwork: clinical correlation in patients with primary open-angle glaucoma. Arch. Ophthalmol. 123: 458-463.

Silva RE, Arruda JT, Rodrigues FW and Moura KK (2009). Primary open angle glaucoma was not found to be associated with p53 codon 72 polymorphism in a Brazilian cohort. Genet. Mol. Res. 8: 268-272.

Sprenger R, Schlagenhaufer R, Kerb R, Bruhn C, et al. (2000). Characterization of the glutathione S-transferase GSTT1 deletion: discrimination of all genotypes by polymerase chain reaction indicates a trimodular genotype-phenotype correlation. Pharmacogenetics 10: 557-565.

Ünal M, Guven M, Devranoglu K, Ozaydin A, et al. (2007). Glutathione S transferase M1 and T1 genetic polymorphisms are related to the risk of primary open-angle glaucoma: a study in a Turkish population. Br. J. Ophthalmol. 91: 527-530.

Yang J, Tezel G, Patil RV, Romano C, et al. (2001). Serum autoantibody against glutathione S-transferase in patients with glaucoma. Invest. Ophthalmol. Vis. Sci. 42: 1273-1276.

Yildirim O, Ates NA, Tamer L, Oz O, et al. (2005). May glutathione S-transferase M1 positive genotype afford protection against primary open-angle glaucoma? Graefes Arch. Clin. Exp. Ophthalmol. 243: 327-333. 